\title{
Output Track Controller with Gravitational Compensation for a Class of Hyper-Redundant Robot Arms
}

\author{
Mihaela FLORESCU, Van Dong Hai NGUYEN, Mircea IVANESCU \\ University of Craiova, 13 Al. I. Cuza Street, Craiova, 200585, Romania, \\ mihaelaflorescu@yahoo.com, florescu.mihaela@ucv.ro, \\ donghai.spkt@gmail.com, ivanescu@robotics.ucv.ro
}

\begin{abstract}
The paper studies the output tracking control problem of a class of hyper-redundant robotic arms described by hyperbolic equations. The stability analysis and the resulting controllers are obtained by using the concept of boundary geometric control and an output tracking technique. A conventional PD control is proposed and analysed. Then, for a dynamic model with uncertain components, a robust algorithm is discussed. The output stability is analysed. Numerical simulations are also provided to verify the effectiveness of the approach presented.
\end{abstract}

Keywords: Hyper-redundant arm, gravitational compensation, controller.

\section{Introduction}

The goal of this paper is to implement a control system for a class of hyper-redundant robots with continuum components. This class of robots represents one of the most attractive domains of robotics during the last decades. In [1-4], were analyzed the kinematic models by the "backbone curve". The papers [5-7] derived a new kinematic model by using the differential geometry, $[8,12]$ studied the manipulability of continuum robots. Cable-driven continuum robot control with variable stiffness was studied in [13]. In [14-16] were studied the kinematics of multi-section continuum robots. Several biomimetic robotic prototypes with undulating actuation have been developed in $[17,18]$. The differential kinematic models of a class of continuum micro-robot for endovascular surgery applications are treated in [19-24]. Other papers [26-28,33] use the assumption that the arm bends with constant curvature and propose new control strategies.

In our paper, the main parameter, the system state, is determined by the position generalised variables. The dynamic model is inferred and the constraints of the state variables and nonliner components are proved. The estimation of gravitational terms is very difficult in a complex motion. For this reason, the gravitational forces are treated as uncertain components that satisfy the inequality constraints. An essential part of designing feedback controllers for these models is designing practical controllers that are implementable. The inequality constraints on the gravitational components allow to introduce a decoupled control system. A PD boundary control algorithm is used in order to achieve a desired shape of the arm. The stability analysis and the resulting controllers are obtained using Liapunov techniques. The exponential stability of the system (error-observer) was proved. Numerical simulations and experimental tests verify the effectiveness of the presented techniques.

The paper is organized as follows. In Section 2, the dynamic model is presented. Section 3 concerns the formulation of the outputfeedback control and the design methodology of a PD output track controller. Section 4 presents the simulation results. Finally, a Conclusion Section ends the article.

\section{Model Description}

The technological model basis is a light weight arm with a distributed mass and friction. Although the conventional hyper-redundant models operate in 3-D space, the motion control will be first infer from the planar models. The 2D model basis from Figure 1 consists of a chain of vertebrae, elements, periodically spaced, each element having a special joint that ensures the rotation, elastic contact and a controllable friction force with the following element. All the joints are passive. All these elements determine a backbone type behavior of the arm. The motion of the arm, the bending, is determined by a pair of antagonistic cables (tendons) attached to the terminal point of the arm and that run through all the elements. The essence of the arm is the backbone curve $\mathrm{C}$ (Figure 2). The independent 
parameter $\mathrm{s}$ is related to the arc-length from origin of the curve C, $s \in \Omega, \Omega=[0, l]$, where 1 is the length of the arm. The position of a point $s$ on curve $C$ is defined by the position vector $r=r(s), s \in[0, l]$. For a dynamic motion, the time variable will be introduced, $r=r(s, t)$. We denote by $q$ the slope of the curve, $q=q(s)$ is the generalized coordinate, where $q \in L_{2}(\Omega)$ and $L_{2}(\Omega)$ is the Hilbert space of square integrable functions $q(s)$, $s \in[0, L]$ equipped with $L_{2}$ norm

$$
\|q(\cdot)\|=\sqrt{\int_{0}^{L} q^{2}(s) d s}
$$

Also, $\tau$ represents the equivalent moment at the end of the arm $(s=l)$ exercised by the cable forces.

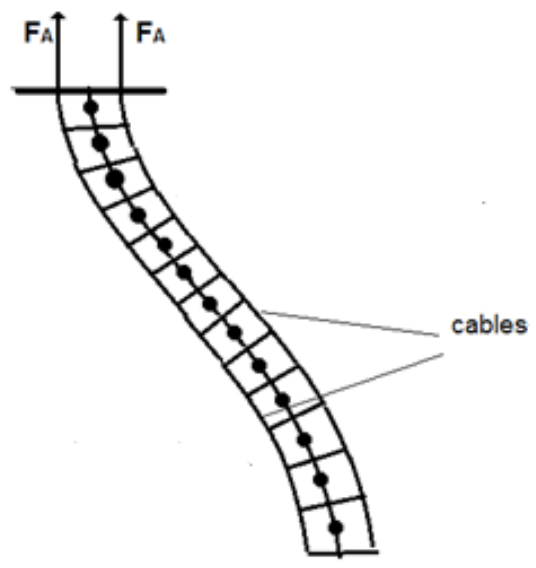

Figure 1. Technological arm

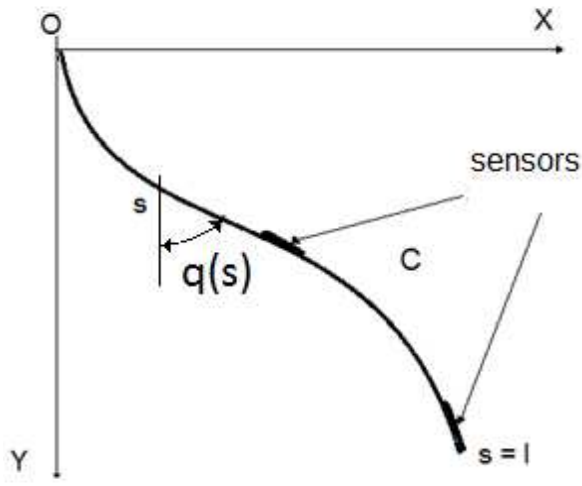

Figure 2. Ideal planar model

The dynamic model of the arm can be derived from the Hamiltonian principle [ ] as

$$
I_{\rho} \ddot{q}=E I q_{s s}-b \dot{q}+c q-h(q)
$$

where $q=q(t, s), s$ is a mono-dimensional (1D) spatial variable, $s \in[0, l], t \geq 0$ is time, $\dot{q}$ represents $(\partial q(t, s)) / \partial t$ and $q_{s}=\partial q(t, s) / \partial s$ ,$I_{\rho}$ is the rotational inertial density, $E I$ is the bending stiffness coefficient, $b$ is the equivalent damping matrix of the arm, $c$ characterizes the elastic behaviour and $h(q)$ represents the nonlinear term determined by gravitational components. The state variables are $q \in L_{2}(0, l), \dot{q} \in L_{2}(0, l)$.

We assume the following initial conditions

$$
\begin{aligned}
& q(0, s)=q_{0}(s) \in H^{2}(0,1) \\
& \dot{q}(0, s)=q_{1}(s) \in H^{2}(0,1)
\end{aligned}
$$

and the boundary conditions

$$
q_{s}(t, 0)=0, E I q_{s}(t, l)=\tau \text {, }
$$

The gravitational component satisfies the inequality [ ]

$$
\|h(q(s))\| \leq \eta\|q\|=\rho g L\|q\|
$$

The output of the system is represented by the weighted average values defined by the relation

$$
y(t)=\int_{0}^{l} w(s) q(t, s) d s, \quad y \in C^{2}(0, l)
$$

where $w(s)$ is spatial weighting measuring function, $w \in L_{2}(0, l)$. We assume that $w$ satisfies the following conditions:
a) $w_{s s}=-\lambda w$
b) $w(s)>0, s \in(0, l)$
c) $w(0)=0 ; w_{s}=0$

where $\lambda$ is a positive constant.

\section{A PD Output-Feedback Control}

We consider a desired state $q^{d}(s)$, $q^{d} \in L_{2}(0, l)$ that satisfies the Eq. (2.1) with initial and boundary conditions (2.2), (2.3).

A desired output can be defined as

$$
y^{d}(t)=\int_{0}^{l} w(s) q^{d}(t, s) d s
$$

The control problem consists in the finding the control law $\tau(t)$, on the boundary $s=l$, such that the output $y(t)$ to track the "a priori" given desired output $y^{d}(t)$.

Definition 1. The control system is stable if

$\lim _{t \rightarrow \infty}(t)=y^{d}(t)$

In terms of this definition we can synthesize a PD (Proportional - Derivative) output-feedback controller that enforce output tracking and guarantee stability in the closed loop system (Figure 3). 


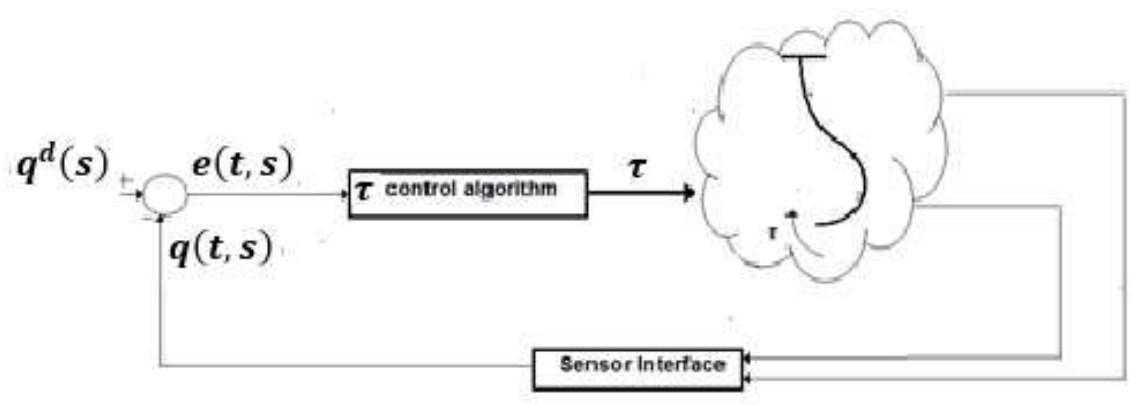

Figure 3. Control system

Theorem 1. An output - feedback control of the system $(2.1)-(2.3)$ is stable, in the sense of Definition 1, if the control law is

$$
\begin{aligned}
\Delta \tau(t) & =-(E I) w_{s}(0)\left(q(t, 0)-q^{d}(t, 0)\right)- \\
& -k_{1} \int_{0}^{l} w(s)\left(q(t, s)-q^{d}(s)\right) d s- \\
& -k_{2} \int_{0}^{l} w(s) \dot{q}(t, s) d s
\end{aligned}
$$

where $k_{1}, k_{2}$ are the control coefficients, $k_{1}>0, k_{2}>0$

$k_{1}>(c-\lambda E I)+\eta$

$k_{2}>\frac{\gamma}{\beta} \cdot I_{\rho}-b$

$\alpha, \beta$, and $\gamma$ are positive constants that verify the conditions

$$
\begin{aligned}
& \alpha>\gamma \cdot \frac{I_{\rho}}{4}, \beta>\gamma \\
& \alpha+(c-\lambda E I)-\gamma b-\beta k_{1}-\gamma k_{2}+\beta \eta>0 \\
& \Delta \tau(t)=\tau(t)-\tau^{d}
\end{aligned}
$$$$
\tau^{d} \text { is the desired moment applied at } s=l \text {. }
$$

Proof. Denote by $e(t)$ the weighted output error variable

$$
e(t)=\int_{0}^{l} w(s)\left(q(t, s)-q^{d}(s)\right) d s
$$

Substituting (3.8) into (2.1) and integrating by parts,

$q_{s s} w=\frac{\partial}{\partial s}\left(q_{s} w\right)-\frac{\partial}{\partial s}\left(q w_{s}\right)+q w_{s s}$

the error dynamics will be described by

$$
\begin{aligned}
& I_{\rho} \ddot{e}=-b \dot{e}+(c-\lambda E I) e-\Delta h^{*}(e)+ \\
& \quad+\Delta \tau+(E I) w_{s}(0) q(0)
\end{aligned}
$$

where

$$
\Delta h^{*}(e)=\int_{0}^{l} w(s)\left(h(q)-h\left(q^{d}\right)\right) d s
$$

(variables $s, t$ are omitted in order to simplify the notation). From (2.4) can be inferred that

$$
\left\|\Delta h^{*}(e)\right\| \leq \sqrt{2} \eta\|q\|
$$

Consider the following Liapunov function

$$
V(t)=\frac{1}{2}\left(\alpha e^{2}+\beta I_{\rho} \dot{e}^{2}+\gamma I_{\rho} e \ddot{e}\right)
$$

and using the conditions in (3.6), (3.13) is positive definite.

The time derivative of (3.13) will be

$$
\dot{V}(t)=\alpha e \dot{e}+\beta I_{\rho} \dot{e} \ddot{e}+\gamma I_{\rho} \dot{e}^{2}+\gamma I_{\rho} e \ddot{e}
$$

The following inequalities can be obtained by using the gravitational inequality (3.12)

$$
\begin{aligned}
& \left|-\dot{e} \Delta h^{*}(e)\right|<\eta|\dot{e} \| e| \\
& \left|-\dot{e} \Delta h^{*}(e)\right|<\eta e^{2}
\end{aligned}
$$

Now, substituting the error dynamics (3.10) into (3.14), the control law $\Delta \tau(t)$ from (3.3), after simple additional manipulations, it is obtained

$$
\begin{aligned}
\dot{V}(t) & <\left(\alpha+(c-\lambda E I)-\lambda b-\beta k_{1}-\gamma k_{2}+\beta \eta\right)|\dot{e} \| e|- \\
& -\left(\beta b-\gamma I_{\rho}+\beta k_{2}\right) \dot{e}^{2}- \\
& -\gamma\left(k_{1}-(c-\lambda E I)-\eta\right) e^{2}
\end{aligned}
$$

Using the conditions (3.4), (3.5), (3.7), this inequality is negative definite

$$
\dot{V}(t)<0
$$

Remark 1 The control system (3.3) - (3.7) is exponentially stable.

Proof. The Liapunov function (3.13) satisfies the following inequality

$$
V(t)<V^{*}(t)=\frac{M^{*}}{2}\left(e^{2}+\dot{e}^{2}\right)
$$

where $M^{*}=\max \left(\alpha, \beta I_{\rho}\right)$.

The inequality (3.17) can be rewritten as

$$
\dot{V}(t)<-m^{*}\left(e^{2}+\dot{e}^{2}\right)=-\frac{2 m^{*}}{M^{*}} V^{*} \leq-\frac{2 m^{*}}{M^{*}} V(t)
$$


From (3.20) it can be then concluded that the system (3.6)-(3.7) is exponentially stable

$$
V(t) \leq V(0) e^{-2 \frac{m^{*}}{M^{*}} t}
$$

Remark 2 The conditions (3.4), (3.5) can be rewritten as

$$
\begin{aligned}
& k_{1}>c+\eta \\
& k_{2}>\frac{\gamma}{\beta} I_{\rho}
\end{aligned}
$$

Remark 3. The analyze of the motion stability can be obtained by the describing functions associated to the nonlinear components of the gravitational term. The describing functions of the nonlinear gravitational component $\Delta h^{*}$ are represented by the fundamental components with respect to a sinusoidal input $A^{*} \sin \left(\omega^{*} t\right)$, where $A^{*}, \omega^{*}$ are the amplitude and frequency, respectively [35].

$h_{G}=h_{G 1}+j h_{G 2}$

where

$$
\begin{aligned}
& h_{G 1}=\frac{1}{\pi A^{*}} . \\
& \cdot \int_{0}^{2 \pi} \Delta h^{*} A^{*} \sin \left(\omega^{*} t\right) \sin \left(\omega^{*} t\right) d\left(\omega^{*} t\right) \\
& h_{G 1}=\frac{1}{\pi A^{*}} . \\
& \cdot \int_{0}^{2 \pi} \Delta h^{*} A^{*} \sin \left(\omega^{*} t\right) \cos \left(\omega^{*} t\right) d\left(\omega^{*} t\right)
\end{aligned}
$$

The describing functions are computed for the mechanical parameters $I_{\rho}=0.001 \mathrm{~kg} \cdot \mathrm{m}^{2}$, $E I=1.2 \mathrm{~N} \cdot \mathrm{m}^{3}, \quad b=0.06 \mathrm{Nms} / \mathrm{rad} . \quad$ From (3.24) - (3.26) yields,

$$
h_{G}=0.85+j \cdot 0
$$

For a desired position $y^{d}=0$ and the describing function (3.27), the transfer function $\left(s^{*}\right.$ is Laplace variable) of the arm with $\mathrm{n}$ segments can be approximated as

$$
\begin{aligned}
G\left(s^{*}\right) & =\frac{e\left(s^{*}\right)}{\Delta \tau\left(s^{*}\right)} \cong \\
\cong & \frac{1}{I_{\rho} s^{* 2}+b s^{*}+\left(\lambda E I-c+n h_{G 1}\right)}
\end{aligned}
$$

The instability of the arm segment is determined by the elastic component $E I$ and the gravitational component $h_{G 1}$. This instability increases with respect to the number of segments $(n)$ in the structure of the arm. The closed loop transfer function of the controller and the arm segment is evaluated as

$$
G_{S A}\left(s^{*}\right) \cong \frac{k_{1}+k_{2} s^{*}}{I_{\rho} s^{* 2}+B s^{*}+\left(\lambda E I-c+n h_{G 1}\right)}
$$

The polar plots of $G_{S A}\left(j \omega^{*}\right)$ for the 1segment, 2-segment, 3-segment arms, respectively, are represented in Figure 4. Clearly, by virtue of Nyquist stability criterion, the closed-loop system is stable because the number of counterclockwise encirclements of the $(-1,0)$ point is equal to one, the number of poles with positive real parts $[34,35]$.

\section{Numerical Simulations}

Consider the dynamic model of a hyperredundant continuum robotic arm described by (2.1) where the length of the arm is $l=1$, the rotational inertial density is $I_{\rho}=1$, the bending stiffness $E I=1.5$, the equivalent damping coefficient $b=-0.5$ and the elastic coefficient is $c=15$. These constants are scaled to realistic ratios for long thin arm. The initial and

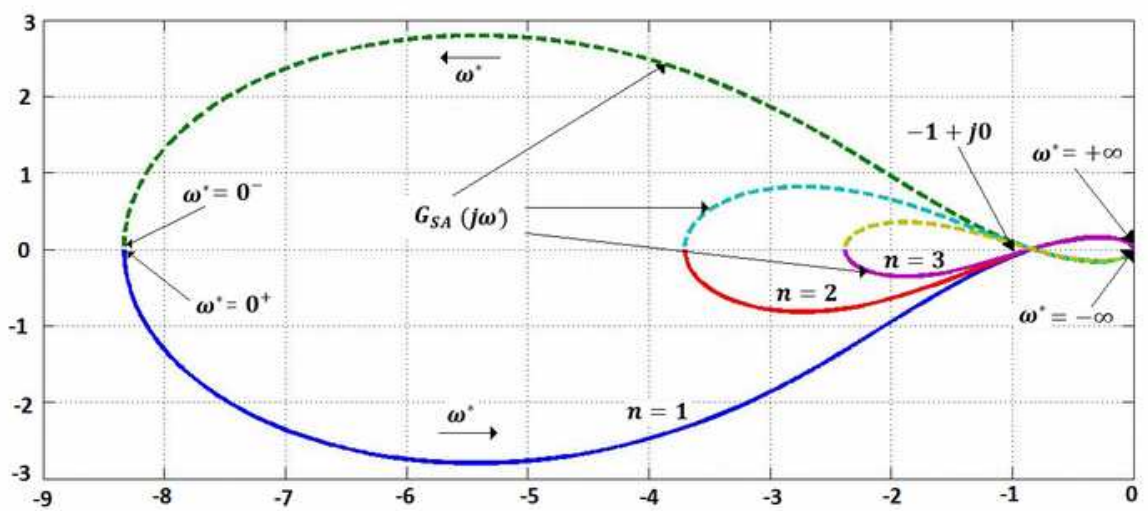

Figure 4. Polar plots of $G_{S A}\left(j \omega^{*}\right)$ for the 1-segment arm, 2-segment arm, 3-segment arm 


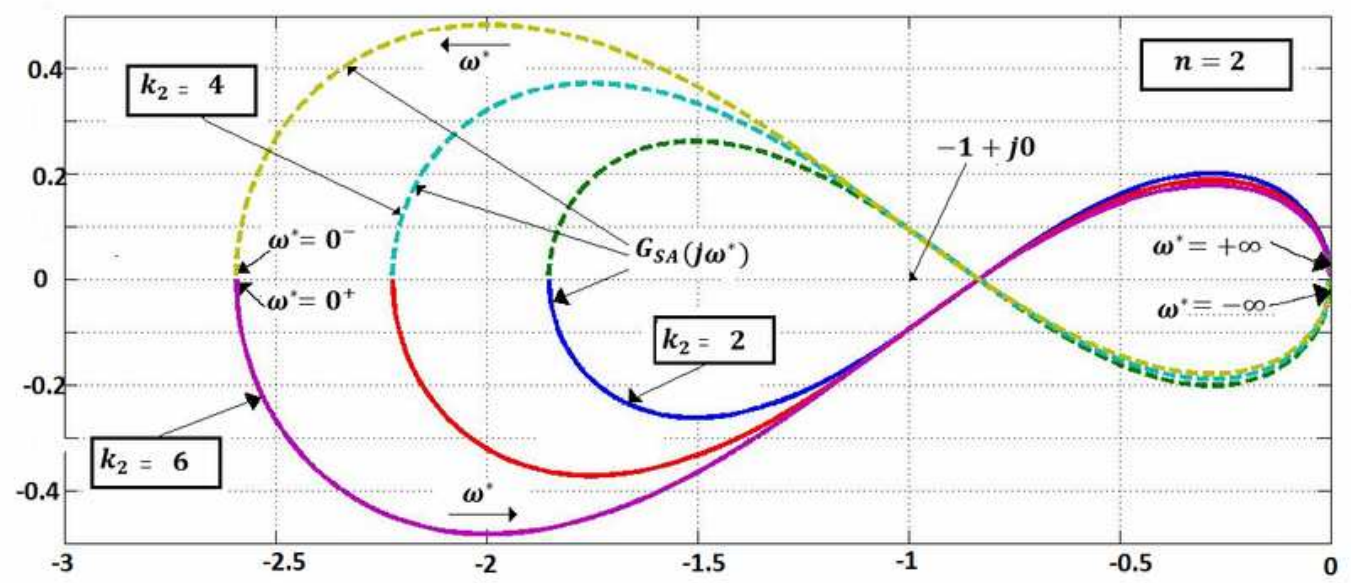

Figure 5. Polar plots of $G_{S A}\left(j \omega^{*}\right)$ for a 2-segment arm (control by $k_{2}$ )

boundary conditions are $q_{0}(s)=0, q_{1}(s)=0$, $q_{s}(t, 0)=0, E I q_{s}(t, l)=\tau$. The desired state is $q^{d}(s)=1.8 \cos (1.5 s)$, that satisfies the stationary desired state for $\tau^{d}=-3.8$.

A spatial weighting measuring function $w(s)=\sin \left(\frac{\pi s}{2}\right)$ that satisfies the conditions
(2.6) for $\lambda=\left(\frac{\pi}{2}\right)^{2}$ and a control law (3.3) with $k_{1}=18, k_{2}=2$ are used. Figure 6 and Figure 7 reports three-dimensional plots of the solution $q(t, s)$ and of the tracking weighted error $e(t)$. Good performances of the proposed control algorithm is concluded from the graphics.

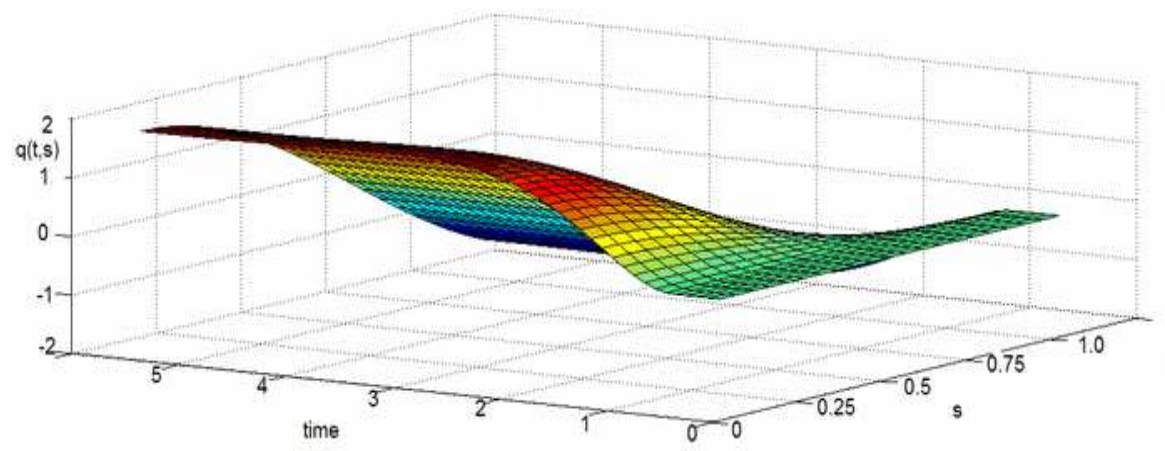

Figure 6. The state evolution $q(t, s)$ for the desired state $q^{d}(s)=1.8 \cos (1.5 s)$

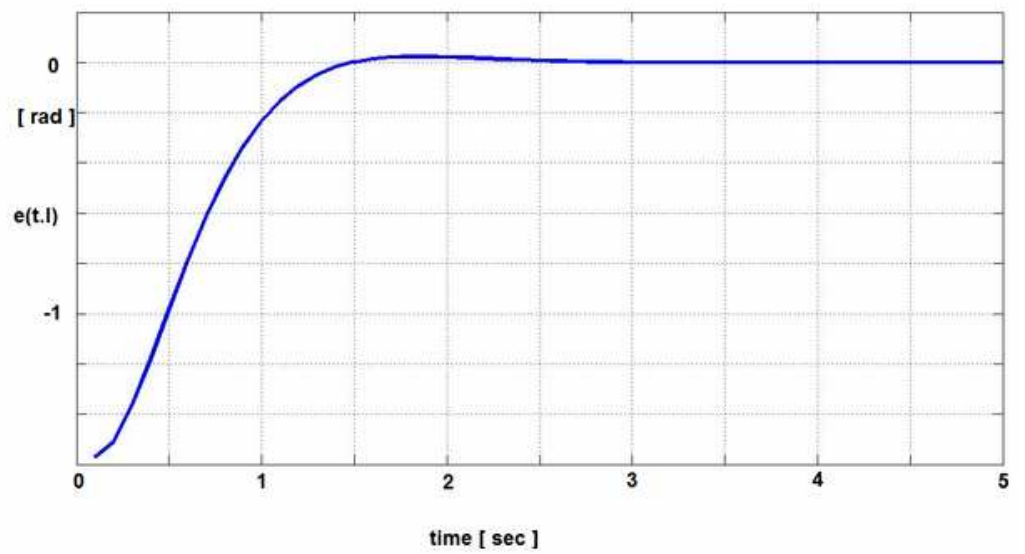

Figure 7. Tracking weighted error $e(t)$ 


\section{Conclusion}

This paper deals with the control problem of a class of robots constituted by a chain of continuum segments. The technological model basis is a central, long and thin, highly flexible and elastic backbone. The paper studies the output tracking control problem of a class of DPS described by hyperbolic DPE. The stability analysis and the resulting controllers are obtained by using the concept of boundary geometric control and an output track technique. A conventional PD control is proposed and analyzed. Numerical simulations are also provided to verify the effectiveness of the presented approach.

\section{Acknowledgement}

This work was supported by the strategic grant POSDRU/159/1.5/S/133255, Project ID 133255 (2014), co-financed by the European Social Fund within the Sectorial Operational Program Human Resources Development 2007 - 2013.

\section{REFERENCES}

1. ROBINSON, G., DAVIES, G.B.C., Continuum Robots - A state of the art, Proceedings of IEEE International Conference on Robotics and Automation, Detroit, May 1999, pp. 2849-2854.

2. GRAVAGNE, I. A., I. D. WALKER, On the Kinematics of Remotely - Actuated Continuum Robots, Proceedings of the 2000 IEEE International Conference on Robotics and Automation, San Francisco, April 2000, pp. 2544-2550.

3. GRAVAGNE, I. A., I. D. WALKER, Kinematic Transformations for Remotely-Actuated Planar Continuum Robots, Proceedings of the 2000 IEEE International Conference on Robotics and Automation, San Francisco, April 2000, pp. 19-26.

4. GRAVAGNE, I. A., I. D. WALKER, Uniform Regulation of a Multi-Section Continuum Manipulators, Proceedings of the 2002 IEEE International Conference on Robotics and Automation, Washington DC, May 2002, pp. 1519-1525.

5. CHIRIKJIAN, G. S., J. W. BURDICK, An Obstacle Avoidance Algorithm for
Hyper-redundant

Manipulators,

Proceedings of the IEEE International Conference on Robotics and Automation, Cincinnati, Ohio, May 1990, pp. 625-631.

6. MOCHIYAMA, H., H. KOBAYASHI, The Shape Jacobian of a Manipulator with Hyper Degrees of Freedom, Proceedings of the 1999 IEEE International Conference on Robotics and Automation, Detroit, May 1999, pp. 2837-2842.

7. JINGLIN, L., JING, X., Determining Grasping Configurations for a Spatial Continuum Manipulator, 2011 IEEE/RSJ International Conference on Intelligent Robots and Systems, September, 25-30, 2011, San Francisco, pp 4207-4213.

8. WALKER, I. D., M. W. HANNAN, The 'Elephant Trunk' Manipulator, Design and Implementation, 1999 IEEE/ASME International Conference on Advanced Intelligent Mechatronics, (AIM 1999), Atlanta, USA, September 19 - 23, 1999, pp. 410-415.

9. JONES, B., I. D. WALKER, Practical Kinematics for Real-time Implementation of Continuum Robots, IEEE Transaction on Robotics, vol. 22, no. 6, Dec. 2006, pp. 1087-1099.

10. KAPADIA, I., I. D. WALKER, D. M. DAWSON, A Model-based Sliding Mode Controller for Extensible Continuum Robots, Recent Advances in Signal Processing, Robotics and Automation, ISPRA Conference, 2009, pp. 103-120.

11. GRAVAGNE, I., I. D. WALKER, Manipulability and Force Ellipsoids for Continuum Robot Manipulators, 2001 IEEE/RSJ International Conference on Intelligent Robots and Systems, Maui, Hawaii, October 29 - 31, pp. 304-310.

12. RUCKER, D. C., R. J. WEBSTER III, G. S. CHIRIKJIAN, N. J. COWAN, Equilibrium Conformations of Concentric-Tube Continuum Robots, International Journal of Robotic Research, 2010, vol. 29, no. 10, pp. 1263-1280.

13. POPESCU, N., D. POPESCU, M. IVANESCU, A Spatial Weight Error Control for a Class of Hyper-redundant Robots, IEEE Transactions on Robotics, ISSN 1552-3098, August 2013, vol. 29, No. 4, pp. 1043-1050. 
14. POPESCU, N., D. POPESCU, M. IVANESCU, $M$. NITULESCU, The Curvature Control of a Hyperredundant Robot, Proceedings of International Symposium of Robotics, Munich, June 2014, pp. 251-257.

15. IVANESCU, M., N. BIZDOACA, M. FLORESCU, N. POPESCU, D. POPESCU, Frequency Criteria for the Grasping Control of a Hyper-redundant Robot, Proceedings of IEEE International Conference on Robotics and Automation, Anchorage, Alaska (ICRA 2010), May 3 8, 2010, pp. 1542-1549.

16. LA SPINA, G., M. SFAKIOTAKIS, D. TSAKIRIS, A. MEMCIASSI, P. DARIO, Polychaete-Like Undulatory Robotic Locomotion in Unstructured Substrates, IEEE Transactions on Robotics, vol. 23, No. 6, February 2007, pp. 1200-1212.

17. NING, K. J., F. WORGOTTER, A Novel Concept for Building a HyperRedundant Chain Robot, IEEE Transactions on Robotics, vol. 25, No. 6, December 2009, pp 1237-1248.

18. RUCKER, D. C., B. A. JONES, R. J. WEBSTER III, A Geometrically Exact Model for Externally Loaded Concentric-Tube Continuum Robots, IEEE Transactions on Robotics, vol. 26, No. 5, October 2010, pp. 769-780.

19. BAILLY, Y., Y. AMIRAT, G. FRIED, Modeling and Control of a Continuum Style Microrobot for Endovascular Surgery, IEEE Transactions on Robotics, vol. 27, No. 5, Oct. 2011, pp. 1024-1030.

20. BAJO, A., N. SIMAAN, KinematicsBased Detection and Localization of Contacts along Multisegment Continuum Robots, IEEE Transactions on Robotics, vol. 28, No 2, April 2012, pp. 291-302.

21. BAJO, A., N. SIMAAN, KinematicsBased Detection and Localization on Contacts along Multisegment Continuum Robots, IEEE Transactions on Robotics, vol. 28, No. 2, April 2012, pp. 291-302.

22. JONES, B. A., I. D. WALKER, Kinematics for Multisection Continuum Robots, IEEE Transactions on Robotics, Vol. 22, No. 1, February 2006, pp. 43-51.
23. TRIVEDI, D., C. D. RAHN, W. M. KIER, I. D. WALKER, Soft Robotics; Biological Inspiration, State of Art and Future Research, Applied Bionics and Biomechanics, vol. 5 number 3, 2008, pp. 99-117.

24. FAREH, R, M. SAAD, Workspace Tracking Trajectory for 7-DOF ANAT Robot using a Hierarchical Control Strategy, 20 $0^{\text {th }}$ Mediterranean Conference on Control \& Automation, Barcelona, Spain, July 3-6, 2012, pp. 122-128.

25. SHANG, H., J. F. FORBES, M. GUAY, Feedback Control of Hyperbolic Distributed Parameter Systems, Chemical Engineering Science, vol. 60, 2005, pp. 969-980.

26. FAHIMI, F., H. ASHRAFIUON, C. NATARAJ, An Improved Inverse Kinematic and Velocity Solution for Spatial Hyper-redundant Robots, IEEE Transactions on Robotics and Automation, vol. 18, no. 1, February 2002, pp. 103-107.

27. SOGA, T., N. OTSUKA., Stability Conditions for Switched Linear Systems with Constant Input via Switched Observer, Studies in Informatics and Control, vol. 22(1), 2013, pp. 146-158.

28. ROGELIO, P. V., C. V. CARLOS, On-line Master/Slave Robot System Synchronization with Obstacle Avoidance, Studies in Informatics and Control, vol. 21(1), 2012, pp. 233-246.

29. CHOSET, H., K. M. LYNCH, S. HUTCHINSON, S. G. KANTOR, W. BURGARD, L. E. KAVRAKI, S. THRUN, Principles of Robot Motion, Theory, Algorithms and Implementations, MIT Press, 2005.

30. MA, S., N. TADOKORO, K. INOUE, Influence of Gradient of a Slope to Optimal Locomotion Curves of a Snakelike Robot, International Journal of Advanced Robotics, vol. 20, issue. 4, 2006, pp. 413-428.

31. WEBSTER, R. J., B. A. JONES, Design and kinematic modelling of Constant Curvature Continuum Robots: A Review, The International Journal of Robotics Research, vol. 29, issue 13, 2010, pp. 1661-1683. 
32. HANNAN, M. W., I. D. WALKER, Realtime Estimation for Continuum Robots Using Vision, Robotica, vol. 23, Issue 05, Sept. 2005, pp. 645-661.

33. EDUARDO, G. H., A. B. EDUARDO, Decentralized Formation Control of Multi-agent Robots Systems based on Formation Graphs, Studies in Informatics and Control, vol. 21(1), 2012, pp 124-138.
34. SLOtine, J. J., W. LI, Applied Nonlinear Control, Prentice Hall, 1991.

35. DORF, R. C., Modern Control Systems, Addison - Wesley Publishing, 1992. 